\title{
The comparison of curcumin and nanocurcumin effects on the expression of $E 6$ and $E 7$ human papilloma virus oncogenes and P53 and pRb factors in HeLa and fibroblast cell lines
}

\section{Rezvaneh Vahedian Sadeghi \\ IAU: Islamic Azad University}

Masoud Parsania

Islamic Azad University

Majid Sadeghizadeh

Tarbiat Modares University Faculty of Biological Sciences

Setareh Haghighat

Azad University: Islamic Azad University

Seyedeh Sahar Mortazavi Farsani ( $\nabla$ sahar.mortazavi66@gmail.com )

Tarbiat Modares University Faculty of Biological Sciences https://orcid.org/0000-0002-8705-1569

\section{Research Article}

Keywords: Cervical cancer, Papillomavirus, Curcumin, Nanocurcumin

Posted Date: March 2nd, 2021

DOI: https://doi.org/10.21203/rs.3.rs-254954/v1

License: (c) (1) This work is licensed under a Creative Commons Attribution 4.0 International License.

Read Full License 


\section{Abstract}

Cervical cancer is a common cancer in women around the world. Human papillomavirus infection is main cause of this cancer. Currently treatment methods include chemotherapy, radiation therapy and surgery. These methods usually cause severe reactions and drug resistance in the patients, so finding a safe natural drug for treatment can be very helpful. Curcumin is a compound derived from the root of the Curcuma longa plant and is the active ingredient in a food spice. Various studies have confirmed the anticancer, chemoprotective and genes and proteins regulatory effects of this component. Insolubility, low cellular uptake and low bioavailability are common limitations of curcumin use. To overcome these limitations, a nano-formulation of curcumin has been developed in our laboratory. In this study, the effect of nanocurcumin on cellular apoptosis induction of Hela cancer cells was compared with curcumin. It has demonstrated that nanocurcumin function is three time more effective than curcumin in same concentration. This nano-formulation of curcumin in $15 \mu \mathrm{M}$ concentration was able to reduce the expression of $E 6$ and $E 7$ oncogenes, and increase $P 53$ and $R b$ tumor suppressors in HeLa cancerous cells with $p$-value $=0.001-0.0001$, whereas curcumin altered these genes expression in $50 \mu \mathrm{M}$ concentration with $p$-value $=0.05-0.01$. Nanocurcumin has no significant effect on the viability of normal fibroblast cells. Therefore, this compound has no effect on normal cells. These findings are consistent with the results of previous studies about nanocurcurcumin in other types of cancer. Therefore, nanocurcumin may be a suitable option to consider for cervical cancer treatment.

\section{Introduction}

Cervical cancer is the third most common cancer in women. 569,000 new cases and 311,000 deaths from this cancer were reported in 2018 [1]. Important risk factors for this cancer include human papillomavirus (HPV), smoking, and immune disorders [2]. HPV is the most critical factor in the development of cervical cancer and HPV test is performing for screening of cervical cancer $[3,4]$.

Carcinogenic HPV types (such as HPV16 and HPV18) target cervical epithelial cells and are responsible for almost all cases of cervical cancer [5].

The human papillomavirus genome can encode eight genes include $L 1, L 2, E 1, E 2, E 4, E 5, E 6$ and $E 7$ [6]. Among these genes, $E 6$ and $E 7$ are the main oncogenic factors of this virus. These factors target different cellular proteins. E6 protein can bind to P53 tumor suppressor and inactive it. E7 protein can disrupt the $\mathrm{pRb}$, terminates their association with the E2F transcription factor family and destroys cell cycle regularity [7]. In the vast majority of human tumors, the functions of pRb and p53 are impaired. Decreased level of $R b$ gene leads to disruption of cell proliferation regulation and apoptosis. P53 gene reduction also eliminates the sensitivity of cells to checkpoint and apoptosis signals [8]. In cervical cancer, the expression of E6 and E7 genes increase in the full-thickness epithelial lesion $[9,10]$. In this study, the effect of nanocurcumin on the $E 7, R b$ and $P 53$ expression in RNA and protein levels was investigated in HeLa cancer cell line. HeLa cells are immortal cells taken from a woman with cervical cancer. HPV infection in these cells is an important cause of cancer progression. HPV virus genome can 
integrate into the host cell genome and express viral oncogenes. Two HPV oncogenes, E6 and E7, are expressed in cervical carcinomas and carcinoma-derived cell lines such as HeLa.

Curcumin, a safe and well-known natural compound, has attracted the attention of researches. This compound is derived from the rhizomes of the Curcuma longa and use as nutritional supplement. Curcumin affects many of cellular pathways. So far, various applications of this compound have been reported, such as inducing cell differentiation[11,12] and anti-cancer potential by regulating many proliferative, oncogenic, and chemo-resistance associated genes or proteins. [13]. The effect of curcumin on cervical cancer cells and its anti-HIV property have been determined [14], but nano-formulation of curcumin containing OA400 nano-carrier has not been evaluated in cervical cancer so far. Curcumin can arrest the proliferation of cervical cancer cells depending on the time and concentration and it is more active in HPV-infected cells. The anti-cancer activity of curcumin against cervical cells was due to regulation of telomerase activity, Ras and ERK signaling pathways, cyclin D1, COX-2 and iNOS activity and mitochondrial pathway. Recent proteomic studies have shown that curcumin causes significant changes in proteins associated with cell metabolism, cell cycle, and carcinogenesis in HeLa cells. The decreasing of cervical tumor volume has also been demonstrated in the curcumin-treated mouse model [15].

Due to the fact that void curcumin has low bioviability, low cellular absorption and insolubility in water, a nano-formulation of curcumin was developed in Professor Sadeghizadeh laboratory to overcome these limitations. A nano-carrier contain oleic acid and PEG400 was used in this carrier [16].

In this study, the reduction of cell viability and apoptosis induction were determined in HeLa cells that treated with nanocurcumin. Interestingly these results were not observed for fibroblast cells. Gene and protein expression analysis were shown the upregulation of P53 and pRb factors and downregulation of E6 and E7 under the influence of nanocurcumin treatment. These data indicate the potential of nanocurcumin to consider as an ameliorative agent in cervical cancer investigations.

\section{Material And Methods}

\section{Nanocurcumin preparation}

The method of preparation of nanocurcumin has been stated in previous studies in the prof. Sadeghizadeh laboratory [17]. Briefly, OA400 nanocarrier was fabricated during the estrification reaction between oleyl chloride (purchased from SigmaAldrich Company) and polyethylene glycol 400 (Merck Company) in the presence of triethylamine (Merck Company) and chloroform (Merck Company) as the solvent at 25खC. Appropriate concentrations of curcumin (purchased from SigmaAldrich Company) and nanocarrier $(1: 25 \mathrm{w} / \mathrm{w})$ were mixed together in order to nanocurcumin preparation.

\section{Cell culture and reagents}


The human cervical cancer cell line (HeLa cell line RRID:CVCL_003) and fibroblast normal cells were purchased from the Pasteur Institute of Iran. DMEM growth medium contains $10 \%$ (v:v) fetal bovine serum (FBS) and 1\% (v:v) of penicillin and streptomycin (all from GibcoBioCult, Paisley, Scotland, UK) used for the culture of HeLa and fibroblast cells. Incubation condition consisted of $\mathrm{CO} 2(5 \%)$ at $37^{\circ} \mathrm{C}$. Cell number and viabilities were defined by hemocytometer and trypan blue staining. These cells were passaged via trypsin/EDTA (biosera, Franc).

\section{MTT assay}

The effects of nanocurcumin, curcumin and OA400 in HeLa and fibroblast cells viability were detected via MTT (3-(4,5- dimethylthiazol-2-yl)-2,5-diphenyltetrazolium bromide) assay. Briefly at first the number of cells was measured by hemocytometer using trypan blue staining. Approximately $10^{4}$ cells were seeded in each well of 96 well-plate, after incubation for 24 hours, cellular monolayers were formed, then treatment with nanocurcumin $(10 \mu \mathrm{M}-40 \mu \mathrm{M})$, curcumin $(10 \mu \mathrm{M}-70 \mu \mathrm{M})$ and OA400 $(10 \mu \mathrm{M}-160 \mu \mathrm{M})$ were performed for 48 hours. Due to the fact that cancer cells are more sensitive to curcumin in compare with normal cells [18], fibroblast cells were treated with higher concentrations of this compound (shown in the results section). After the time required for treatment, the medium of each well was replaced with a solution containing MTT $\left(0.5 \mathrm{mg} \mathrm{ml}^{-1}\right)$ (Sigma Aldrich Company) and incubated for $4 \mathrm{~h}$ at $37^{\circ} \mathrm{C}$ in $5 \%$ of CO2. Formazan was dissolved using 200 $\mu$ l of dimethyl sulfoxide (DMSO from Sigma Aldrich Company) and optical density (OD) of samples were measured at $540 \mathrm{~nm}$ by plate Reader (BioTek Company, USA). Cell survival percentage was obtained by the absorption ratio of the treated cells to the control cells. The concentration at which $50 \%$ of the cells were destroyed considered as inhibitory concentration (IC50).

\section{Cellular apoptosis analysis via flowcytometry}

In the apoptosis assay, HeLa and fibroblast cells were treated with curcumin $(50 \mu \mathrm{M})$ and nanocurcumin $(15 \mu \mathrm{M})$ according to IC50 value. After 48 hours cells were stained with Annexin V/ PI (propidium iodide) apoptosis detection kit according to the manufacturer's instruction (Ebioscience, Thermo Fisher Company). Apoptotic and necrosis levels of cells were detected via flow cytometry (FACS Calibur, USA). Data were subsequently qualified by FlowJo software (version10, Treestar, USA).

\section{RNA extraction, cDNA synthesis and real time RT-PCR}

Total RNA was extracted from treated and untreated HeLa and fibroblast cells with curcumin and nanocurcumin using TRIzol reagent (Invitrogen, Cat.no: 15596-026). Extraction steps were performed according to the instructions of Favorgen Biotech kit (Favorgen Biotech Company, Taiwan) Extracted RNAs were qualified by UV spectrophotometry.

RNA was reverse transcribed using cDNA synthesis kit (Favorgen Biotech Company, Taiwan) according to its manufacture. DNasel enzyme treatment was performed to ensure that RNA was not contaminated with DNA. 
Quantitative measurement of $E 6, E 7, P 53$ and $R b$ genes expressions was performed based on the comparative quantification method by real time RT-PCR in ABI Step One Sequence Detection System (Applied Bio- systems, CA, USA). In this method, SYBR® Premix Ex Taq ${ }^{\text {TM }}$ II (TAKARA, Japan; Cat.RR82LR) was applied according to its manufacture and GAPDH gene utilized as an internal control. Specific primers sequences of target genes have shown in table 1 and their concentrations were $10 \mathrm{pM}$ in the reaction. $2^{-\Delta \Delta C t}$ method was utilized to calculate he relative expression of genes. GraphPad Prism (version 7) software was determined the significance of the results.

\begin{tabular}{|c|c|c|}
\hline Gene & Forward primer & Reverse primer \\
\hline E7 & 5'- CAATTAAGCGACTCAGAGGAAG-3' & 5'- ACCACGGACACACAAAGG-3' \\
\hline$R b$ & 5'- CAGATGAAGCAGATGGAAGTAAA-3' & 5'- AGAGACAATGAATCCAGAGGTG-3' \\
\hline E6 & 5'- GCGACCCTACAAGCTACCTGAT-3' & 5'-GCACCGCAGGCACCTTATTA -3' \\
\hline P53 & 5'- GCCGCAGTCAGATCCTA-3' & 5'- CTGGGAGCTTCATCTGGA-3' \\
\hline GAPDH & 5'- CCGAGCCACATCGCACAG-3' & 5'- GGCAACAATATCCACTTTACCAG-3' \\
\hline
\end{tabular}

\section{Western blotting Analysis}

The protein level of E7, P53 and pRb were determined in curcumin $(15 \mu \mathrm{M})$ and nanocurcumin $(15 \mu \mathrm{M})$ treated HeLa cells, and control group in 48 hours via by Western blotting. In order to protein extraction, the cells were lysed in RIPA buffer (Cell Signaling Technology, Cat No: 9803). This buffer contains $20 \mathrm{mM}$ Tris- $\mathrm{HCl}$ (pH 7.5), $150 \mathrm{mM} \mathrm{NaCl}, 1$ mM EGTA, 1\% NP-40, 1\% sodium deoxycholate. Quantitation of extracted protein was determined by Bradford staining and flowcytometry. An equal amount of proteins samples $(40 \mu \mathrm{g})$ were harmed at $90^{\circ} \mathrm{C}$ for $5 \mathrm{~min}$ and subjected to SDS-PAGE and transferred onto polyvinylidene difluoride filter membranes (PVDF). The BSA (bovine serum albumin)-blocked membranes were incubated with specific primary antibody for E7, P53 and pRb proteins (Mouse monoclonal antibodies, prepared from Royan Institute of Iran) at $4^{\circ} \mathrm{C}$ for overnight according to manufacture instructions. Horseradish peroxidase conjugated secondary antibody (HRP Rabbit Anti-Mouse (IgG) secondary antibody purchased from Abcam Company) employed for the attach to primary antibodies with 1:1000 v:v concentration according to its guideline sheet. Protein bands were detected with enhances chemiluminescence ( $E C L)$ method (Biomedical Company). The internal control for this experiment was $\beta$-Actin protein. This protein was detected by mouse $\beta$-actin primary antibody (1:1000, Abcam Company) and antimouse HRC conjugated secondary antibody (1:1000) (from Santa Cruz). Western blot results were qualified via Image j software (version 1.2.4 RRID:SCR_003070) and significance of the results was indicated by GraphPad Prism (version 7 RRID:SCR_002798).

\section{Statistical analyses}


Student t-test was used for deference examination between two groups. Statistical analyses were performed by GraphPad Prism 7 software. Probability values were set at the significant levels of $p \otimes 0.05$ $\left(p \otimes 0.05\left(^{*}\right), p \otimes 0.01\left(^{* *}\right)\right.$ and $\left.p \otimes 0.001{ }^{(* \star *}\right)$.

\section{Results}

\section{Curcumin and nanocurcumin effect on HeLa and fibroblast cells viability}

MTT calorimetric assay is a method that determine differences between viable cells and necrotic ones [19]. The viability of Hela and Fibroblast cells under treatment with nanocurcumin, pure curcumin and nanocarrier at different concentrations was evaluated by MTT assay for 48 hours.

MTT results of nanocurcumin, curcumin and nanocarrier treatments with different concentrations on the survival of Hela cell line is shown in Fig. 1. As shown in this figure in cancerous HeLa cell line the increasing concentrations of nanocurcumin $(10-40 \mu \mathrm{M})$ in the media can decrease the percentage of viable cells significantly after 48 hours. The IC50 value of nanocurcumin for Hela cell line within 48 hours was about $15 \mu \mathrm{M}$ (Fig. 1A). This growth inhibitory effect of curcumin is much less than nanocurcumin. The value of IC50 for HeLa cells treated with curcumin for 48 hours has been increased to about $50 \mu \mathrm{M}$ (Fig. 1B). OA400 nanocarrier has no inhibitory effect on Hela cell growth. In this experiment, cells were treated with nanocarrier up to $160 \mu \mathrm{M}$, but at none of the concentrations cell survival reached to $50 \%$ (Fig. 1C).

Interestingly, nanocurcumin has not-significant effect on the growth of normal fibroblast cells. The IC50 value was observed at very high concentrations (about $160 \mu \mathrm{M}, \mathrm{Fig} .2 \mathrm{~A}$ ). Pure curcumin and nanocarrier also had no significant inhibitory effect on fibroblast cell growth (Fig. 2B,C).

\section{Nanocurcumin induce apoptosis in cancerous HeLa cells but not in normal fibroblast cells}

Anxin-PI staining and flow cytometry was accomplished to evaluate the effect of nanocurcumin and curcumin on HeLa and fibroblast cells apoptosis. The results of flowcytometry have shown that 46.5 percentages of HeLa cells that treated by $15 \mu \mathrm{M}$ nanocurcumin destructed through apoptosis process. Free curcumin with higher concentration $(50 \mu \mathrm{M})$ can induce apoptosis of $25.9 \%$ of cells. Thus, nanocurcumin is much more effective than curcumin (Fig. 3A). The apoptosis test for fibroblast cells has demonstrated that nanocurcumin $(15 \mu \mathrm{M})$ and curcumin $(50 \mu \mathrm{M})$ had no significant effect on the cellular apoptosis of these normal cells (Fig. 3B).

\section{Nanocurcumin regulation effect on $E 6, E 7, P 53$ and $R b$ mRNA expression}

The expression of $E 6$ and $E 7$ human papilloma virus genes and two related genes include $P 53$ and $R b$ was measured in HeLa cells. Due to the absence of $E 6$ and $E 7$ oncogenes in fibroblasts, expression of $P 53$ and $R b$ was measured in these cells under curcumin and nanocurcumin treatment. The results showed that in HeLa cells, E6 and $E 7$ expression decrease after $48 \mathrm{~h}$ of curcumin and nanocurcumin treatment, whereas $P 53$ and $R b$ expression were enhance after this time in mRNA level with p-value $<0.05$ 
(Fig. 4A,B). The alteration of these genes expression was more significant in nanocurcumin treatment condition. These findings have demonstrated the positive regulation effect of nanocurcumin towards the apoptosis of cancer cells. In fibroblast cells that treated by nanocurcumin, the expression of $p 53$ and $R b$ genes did not altered significantly (Fig. 4C). Therefore, nanocurcumin does not lead normal fibroblast cells to apoptosis process. This result is consistent with findings from MTT and apoptosis assays.

\section{Nanocurcumin regulates E7, P53 and pRb proteins level}

The expression of E7, P53 and phosphorylated Rb proteins were analyzed via western blot assay. The results showed that E7 protein level was reduced in nanocurcumin-treated Hela cancer cells after 48 hours ( $p$-value $<0.05$ ). The phosphorylated Rb and P53 proteins were increased in these cells significantly (p-value < 0.05) (Fig. 5). Western blot results confirm the findings of the real-time RT-PCR assay.

\section{Discussion}

Cervical cancer is a common cancer in women. Many types of HPV virus infection cause about all cases of cervical cancer [5]. Due to the high prevalence of cervical cancer, drug resistance and systemic toxicity for conventional chemotherapy and radiation therapy, it is necessary to identify safe, effective and chemopreventive anti-cancer natural compounds. In this study, the positive regulation effects of nanocurcumin drug on the apoptosis and expression of E6 and E7 oncogenes and their associated tumor suppressor genes include P53 and pRb in human cervical cancer cell line HeLa cells were determined. HeLa cells are derived from the aggressive glandular cervical cancer of a woman and are used in many studies to evaluate cervical cancer treatment methods [20]. E6 and E7 oncogenes in the HeLa cell line derived from HPV genome. The integration of HPV type 16 or 18 DNA to HeLa cells genome has been proven in previous studies. HPV type 18 transcripts in the 8q24 chromosome region of HeLa cell line [21].

In the present study, it was found that nanocurcumin can increase cellular apoptosis and reduce cell viability in human cervical cancerous HeLa cells. Curcumin is a polyphenol compound derived from the root of the turmeric plant and has particularly function in combating cancer in many of studies. Curcumin is also well known as an anti-inflammatory and chemopreventative agent [22].

Studies in the last three decades about curcumin indicate poor bioavailability, hydrophobicity, poor cellular uptake and rapid metabolism of this component [23]. Various experiments have been performed to solve this problem, many of which have performed nanocarriers [24]. In the nanocurcumin that used for this study, OA400 nanocarrier resolved curcumin restrictions. It seems that the faster absorption of the drug is due to the increased permeability of the cell membrane by this nano-carrier.

Previous studies in Professor Sadeghizadeh's laboratory have developed nanocurcumin, including curcumin and OA400 nano carrier, and examined its anti-cancer properties in various cancers $[17,25,26]$. This nano-formulation of curcumin can affect different cellular pathways, including differentiation, cell proliferation and so on $[12,27]$. 
In this study, the effects of curcumin and nanocurcumin on HeLa cell viability and apoptosis were compared. It was found that nanocurcumin is much more effective than curcumin. This finding has been demonstrated in previous studies for other cancer cells [16] and may be due to the fact that nanocurcumin has higher cellular uptake and biostability than curcumin. HeLa and fibroblasts were treated separately with OA400 nanocarrier. The results have indicated that this carrier has no significant effect on cell apoptosis or cellular viability. The non-toxic effect of this nanocarrier proves its safety.

Nanocurcumin has progressed apoptosis process in Hela cancer cells in this experiment but has no significant effect on normal fibroblast cells in a dose dependent manner. This component at very high concentrations $(160 \mu \mathrm{M})$ reduced fibroblast cell viability by approximately $50 \%$. This concentration is not a functional concentration for studies. Therefore, it can be said that nanocurcumin is safe for normal cells at the concentration being studied. There are several reasons that explain why curcumin does not affect normal cells. For example, scientists have shown that the reduction of intracellular glutathione in cancer cells by butyonine sulfoxamine increases ROS levels and makes these cells more sensitive to curcumin [18]. Curcumin has also target molecules that are more common in cancer cells [28]. In previous studies, the capability of nanocurcumin to induce cancerous and undifferentiated cells apoptosis were detected in time and dose dependent manner, while on normal cells, no significant effects observed [29].

E6 and E7 play a key role in the pathogenesis of HPV. These genes encode proteins that bind to P53 and $\mathrm{pRb}$ tumor suppressors respectively and suppress them. This phenomenon disrupts the cell cycle regulation. Expression of these two viral oncogenes is essential for cervical cancer tumorigenesis [30].

In this study, the expression of E6 and E7 viral genes was significantly reduced in HeLa cells during treatment with nanocurcumin. The expression of two tumor suppressor factors affected by these genes, including $P 53$ and $R b$ was also examined. As expected, following the expression decreasing of $E 6$ and $E 7$ genes, the expression level of $P 53$ and $R b$ tumor suppressors increased both at the RNA and protein level. Thus, nanocurcumin provides a condition for the progression of apoptosis in Hela cancer cells and can be considered as a safe complementary drug for cervical cancer.

Curcumin has been applied in several other studies to apoptosis induction in cervical cancer, for example Zaman and his colleagues have evaluated another type of nanocurcumin (poly(lactic-co-glycolic acid) based curcumin nanoparticle formulation) to treat cervical cancer. In their results, as our findings, nanocurcumin in $20 \mu \mathrm{M}$ and $25 \mu \mathrm{M}$ effectively inhibits the growth of cervical cancer cell lines, abrogating expression of $E 6$ and $E 7$ oncogenes, and leads them to apoptosis [15]. In another study investigators showed that curcumin at concentrations above $40 \mu \mathrm{M}$ induced apoptosis in Hela cancer cells [31]. In present study nanocurcumin with lower concentration $(15 \mu \mathrm{M})$ was functional. It confirms that nanocurcumin works more effectively and has more cellular uptake than curcumin.

The Expression of $E 6$ and $E 7$ oncogenes depends on the availability of the host transcription factors such as AP-1 family. Previous studies have shown that AP-1 activity be reduced via curcumin treatment. Curcumin also promote P53 expression by regulation of histone deacetylation and chromatin remodeling. The induction of HeLa cell apoptosis by curcumin has been mediated by NFKB-P53-caspase3 pathway. 
Curcumin can reduce the phosphorylation of IKBa and P65- NFkB subunit in the HeLa cells [32, 33]. Downregulation of E6/E7 and $P 53$ and $R B$ genes in HeLa cells in present study can explain by these mechanisms.

According to our results nano-formulation of curcumin contain OA400 nano-carrier can suppress HPV oncogenes, restore P53 and pRb proteins and induce apoptosis of human cervical cancerous HeLa cells more that curcumin, therefore this nanostructure of curcumin has significant potential to use as complement drug for cervical treatment. So the improving of anti-proliferative function of curcumin that reported in variety of study about different cancer cells can occur by using of nanocarrier [34].

\section{Declarations}

\section{Funding: The research was supported by Islamic Azad University,Tehran, Iran.}

Conflict of interest: The authors declare that there are no conflicts of interest.

Availability of data and material: Not applicable

Code availability: Not applicable

Ethics approval: Not applicable

Consent to participate: Not applicable

Consent for publication: Not applicable

\section{References}

1. Hull, R., et al., Cervical cancer in low and middle-income countries. Oncology Letters, 2020. 20(3): p. 2058-2074.

2. Waggoner, S., Cervical cancer. The Lancet, 2003. 361(9376): p. Pages 2217-2225.

3. Goodman, A., HPV testing as a screen for cervical cancer. Bmj, 2015. 350.

4. Oyervides-Muñoz, M.A., et al., Understanding the HPV integration and its progression to cervical cancer. Infection, Genetics and Evolution, 2018. 61: p. 134-144.

5. Morgan, E.L., et al., E6-Mediated activation of JNK drives EGFR signalling to promote proliferation and viral oncoprotein expression in cervical cancer. Cell Death \& Differentiation, 2020: p. 1-19.

6. Doorbar, J., Molecular biology of human papillomavirus infection and cervical cancer. Clinical science, 2006. 110(5): p. 525-541. 
7. Yim, E.-K. and J.-S. Park, The role of HPV E6 and E7 oncoproteins in HPV-associated cervical carcinogenesis. Cancer research and treatment: official journal of Korean Cancer Association, 2005. 37(6): p. 319.

8. Hickman, E.S., M.C. Moroni, and K. Helin, The role of $p 53$ and pRB in apoptosis and cancer. Current opinion in genetics \& development, 2002. 12(1): p. 60-66.

9. Münger, K., et al., Biological activities and molecular targets of the human papillomavirus E7 oncoprotein. Oncogene, 2001. 20(54): p. 7888-7898.

10. Mantovani, F. and L. Banks, The human papillomavirus E6 protein and its contribution to malignant progression. Oncogene, 2001. 20(54): p. 7874-7887.

11. Mortazavi Farsani, S., et al., The effects of Nanocurcumin on Expression Induction of Transcription Factors Involved in Hematopoietic Stem Cell Differentiation to Precursor Myeloid Cells. Modares Journal of Biotechnology, 2019. 10(4): p. 601-608.

12. Farsani, S.S.M., et al., Nanocurcumin as a novel stimulator of megakaryopoiesis that ameliorates chemotherapy-induced thrombocytopenia in mice. Life Sciences, 2020: p. 117840.

13. Anand, P., et al., Curcumin and cancer: an "old-age" disease with an "age-old" solution. Cancer letters, 2008. 267(1): p. 133-164.

14. Prusty, B.K. and B.C. Das, Constitutive activation of transcription factor AP-1 in cervical cancer and suppression of human papillomavirus (HPV) transcription and AP-1 activity in HeLa cells by curcumin. International journal of cancer, 2005. 113(6): p. 951-960.

15. Zaman, M.S., et al., Curcumin nanoformulation for cervical cancer treatment. Scientific reports, 2016. 6: p. 20051.

16. TAHMASEBI, B.M., et al., Dendrosomal Nano-curcumin, the Novel Formulation to Improve the Anticancer Properties of Curcumin. 2015.

17. Babaei, E., et al., Dendrosomal curcumin significantly suppresses cancer cell proliferation in vitro and in vivo. International immunopharmacology, 2012. 12(1): p. 226-234.

18. Syng-ai, C., A.L. Kumari, and A. Khar, Effect of curcumin on normal and tumor cells: role of glutathione and bcl-2. Molecular cancer therapeutics, 2004. 3(9): p. 1101-1108.

19. Kumar, P., A. Nagarajan, and P.D. Uchil, Analysis of cell viability by the MTT assay. Cold Spring Harbor Protocols, 2018. 2018(6): p. pdb. prot095505.

20. Masters, J.R., HeLa cells 50 years on: the good, the bad and the ugly. Nature Reviews Cancer, 2002. 2(4): p. 315-319. 
21. Diao, M.-K., et al., Integrated HPV genomes tend to integrate in gene desert areas in the CaSki, HeLa, and SiHa cervical cancer cell lines. Life sciences, 2015. 127: p. 46-52.

22. Duvoix, A., et al., Chemopreventive and therapeutic effects of curcumin. Cancer letters, 2005. 223(2): p. 181-190.

23. Anand, P., et al., Bioavailability of curcumin: problems and promises. Molecular pharmaceutics, 2007. 4(6): p. 807-818.

24. Prasad, S., A.K. Tyagi, and B.B. Aggarwal, Recent developments in delivery, bioavailability, absorption and metabolism of curcumin: the golden pigment from golden spice. Cancer research and treatment: official journal of Korean Cancer Association, 2014. 46(1): p. 2.

25. Alizadeh, A.M., et al., Chemoprevention of azoxymethane-initiated colon cancer in rat by using a novel polymeric nanocarrier-curcumin. European journal of pharmacology, 2012. 689(1-3): p. 226-232.

26. Montazeri, M., et al., Dendrosomal curcumin nanoformulation modulate apoptosis-related genes and protein expression in hepatocarcinoma cell lines. International journal of pharmaceutics, 2016. 509(1-2): p. 244-254.

27. Sadeghzadeh, H., et al., The effects of nanoencapsulated curcumin-Fe304 on proliferation and hTERT gene expression in lung cancer cells. Anti-Cancer Agents in Medicinal Chemistry (Formerly Current Medicinal Chemistry-Anti-Cancer Agents), 2017. 17(10): p. 1363-1373.

28. Sordillo, P.P. and L. Helson, Curcumin and cancer stem cells: curcumin has asymmetrical effects on cancer and normal stem cells. Anticancer Research, 2015. 35(2): p. 599-614.

29. Javidi, M.A., et al., Investigating curcumin potential for diabetes cell therapy, in vitro and in vivo study. Life sciences, 2019. 239: p. 116908.

30. Zur Hausen, H., Papillomaviruses and cancer: from basic studies to clinical application. Nature reviews cancer, 2002. 2(5): p. 342-350.

31. Flowers, L.C., et al., Curcumin suppresses growth of cervical cancer cells. 2005, AACR.

32. Mishra, A. and B.C. Das, Curcumin as an anti-human papillomavirus and anti-cancer compound. Future Oncology, 2015. 11(18): p. 2487-2490.

33. Dang, Y.-P., et al., Curcumin improves the paclitaxel-induced apoptosis of HPV-positive human cervical cancer cells via the NF-KB-p53-caspase-3 pathway. Experimental and therapeutic medicine, 2015. 9(4): $\mathrm{p}$. 1470-1476.

34. Ravindran, J., S. Prasad, and B.B. Aggarwal, Curcumin and cancer cells: how many ways can curry kill tumor cells selectively? The AAPS journal, 2009. 11(3): p. 495-510. 

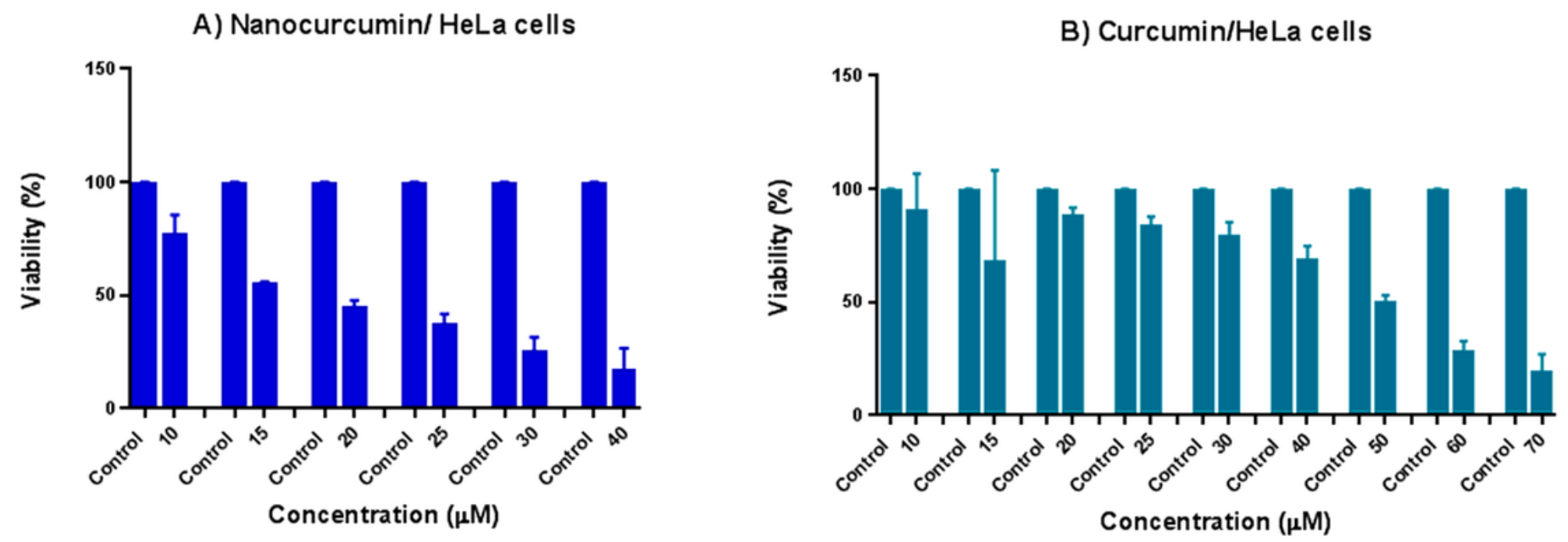

C) NanoCarrier/ HeLa cells

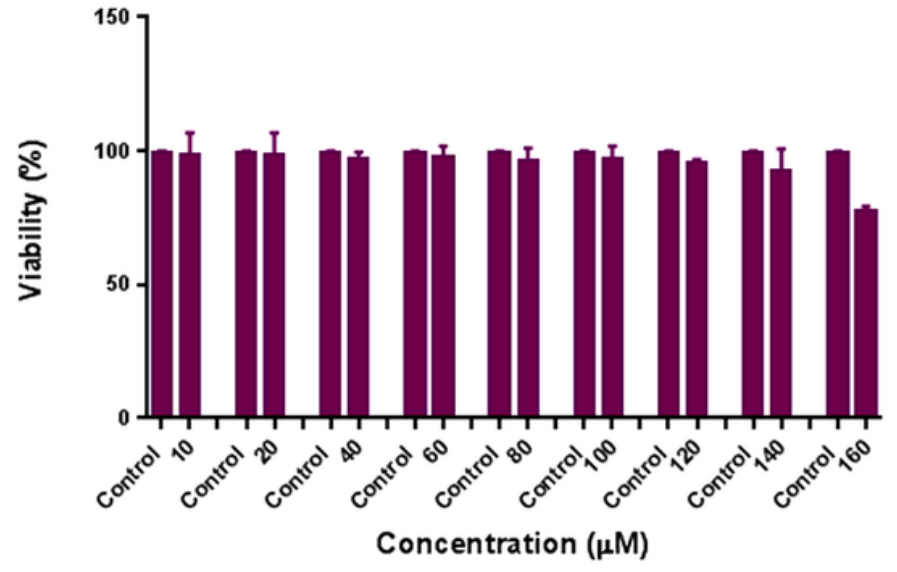

Figure 1

MTT assay result of HeLa cells treated with (A) nanocurcumin, (B) curcumin and (C) nanocarrier. As shown in the figure, nanocurcumin has significant cytotoxicity effect on HeLa cells. Nanocarriers have no side effect for the cells. Error bar lines indicate the standard deviation of samples. The control samples include cells that have not been treated. 
A) Nanocurcumin/ Fibroblast cells

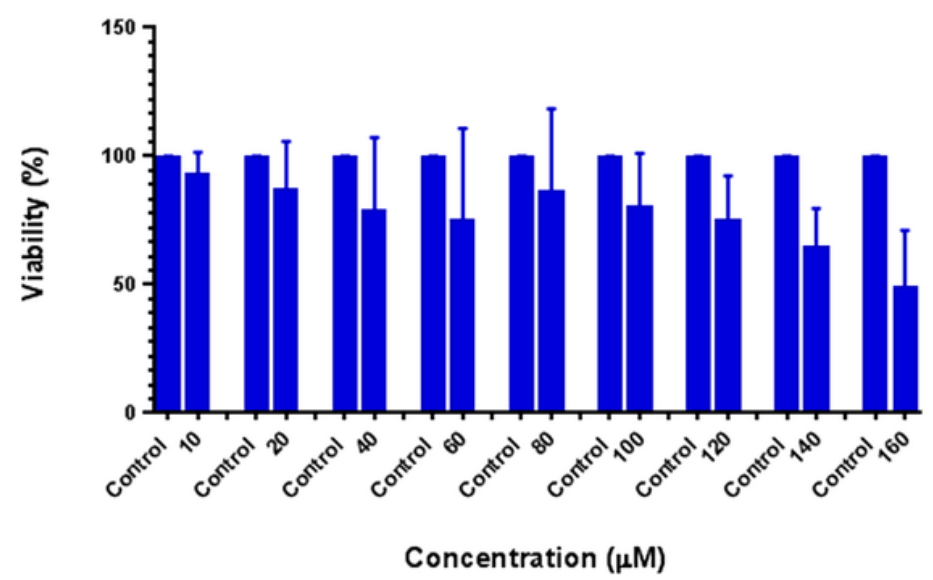

B) Curcumin/Fibroblast cells

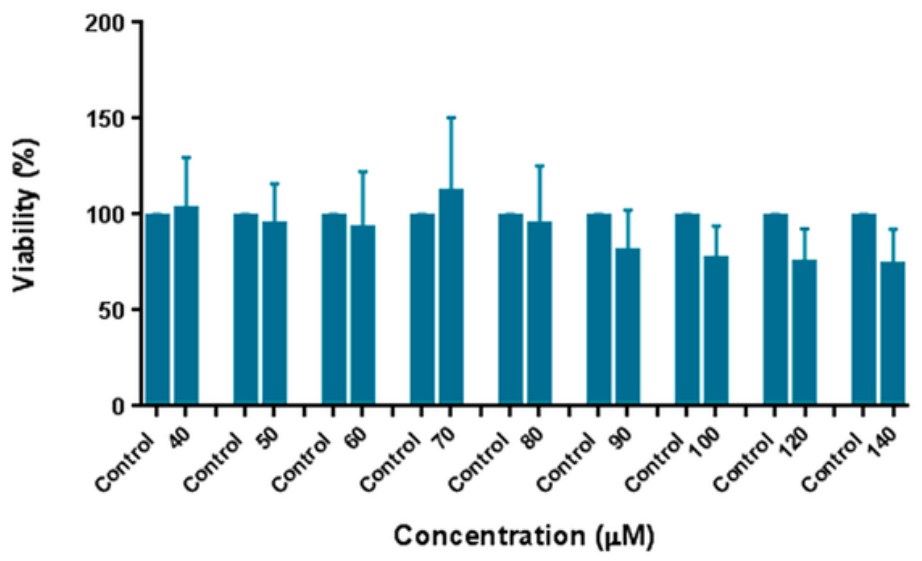

C) NanoCarrier / Fibroblast Cells

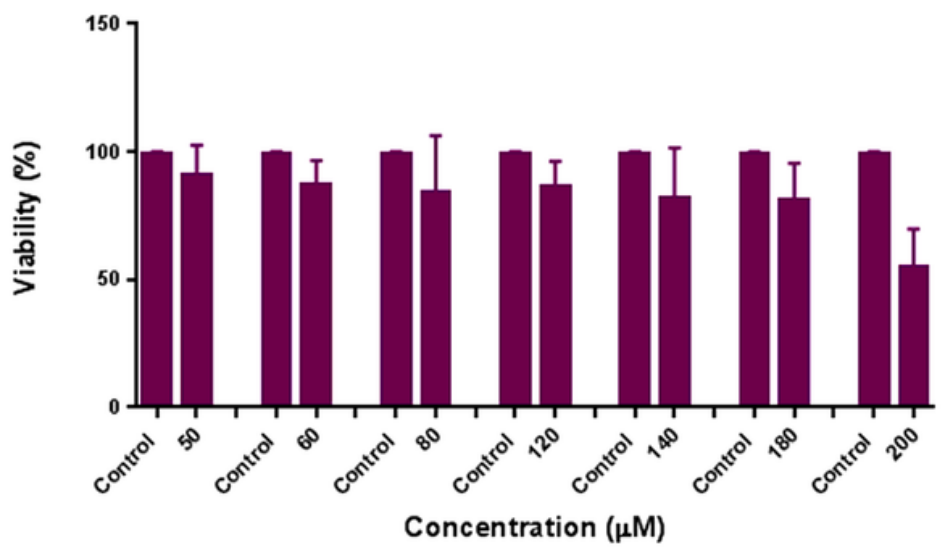

Figure 2

MTT assay result of Fibroblast cells treated with (A) nanocurcumin, (B) curcumin and (C) nanocarrier. Nanocurcumin at very high concentrations $(160 \mu \mathrm{M})$ reduced cell viability by approximately $50 \%$. Neither curcumin nor nanocarrier have been able to reduce cell viability to less than $70 \%$, even at concentrations above $100 \mu \mathrm{M}$. Error bar lines indicate the standard deviation level of samples. The control samples include cells that have not been treated. 


\section{(A) HeLa cells}
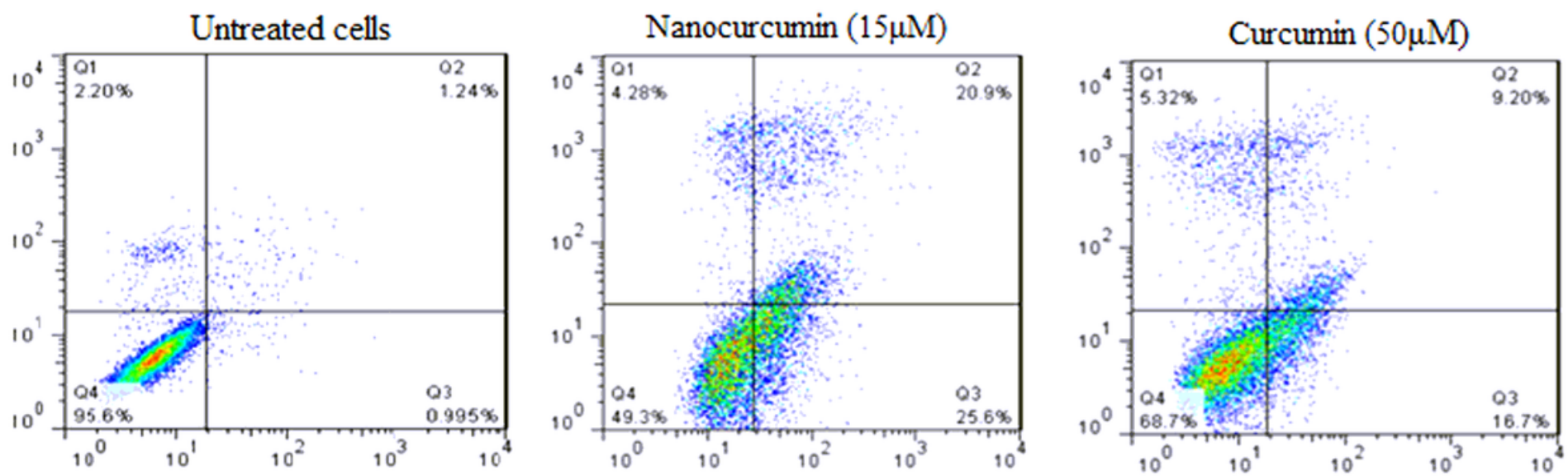

\section{(B) Fibroblast cells}
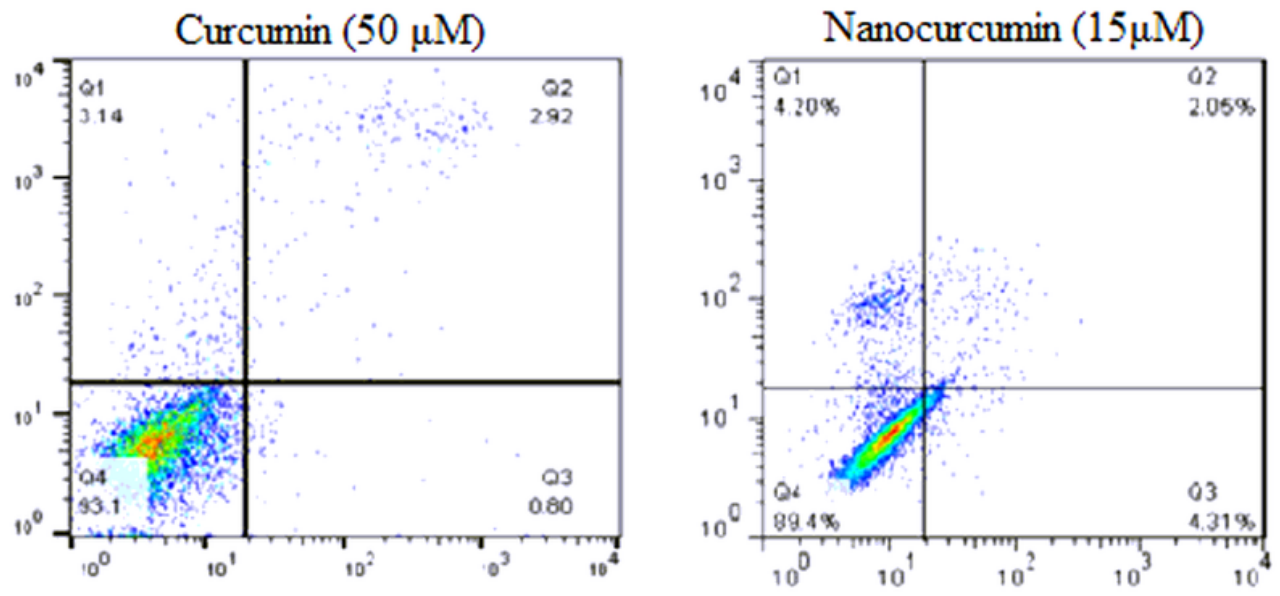

\section{Figure 3}

Flow cytometric diagram obtained from Annexin/PI test to evaluate cellular apoptosis. (A) Apoptosis assay results for cancerous HeLa cells after treatment with $15 \mu \mathrm{m}$ of nanocurcumin and $50 \mu \mathrm{m}$ of curcumin. (B) Apoptosis assay results for fibroblast cells in the same condition. The results have shown that nanocurcumin significantly induces apoptosis in cancer cells, but has no effect on normal cells. 
(A) HeLa cells: P53, E6 expression

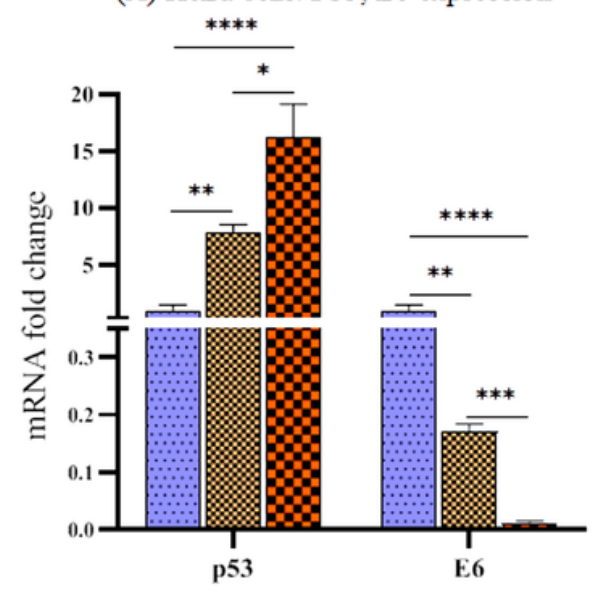

(B) HeLa cells: Rb, E7 expression

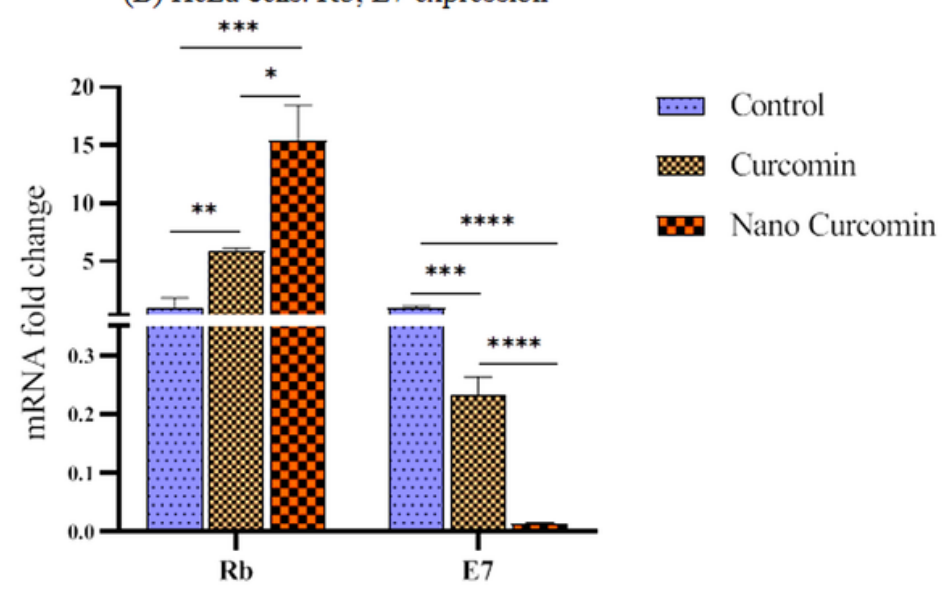

(C) Fibroblast cells, P53, RB expression

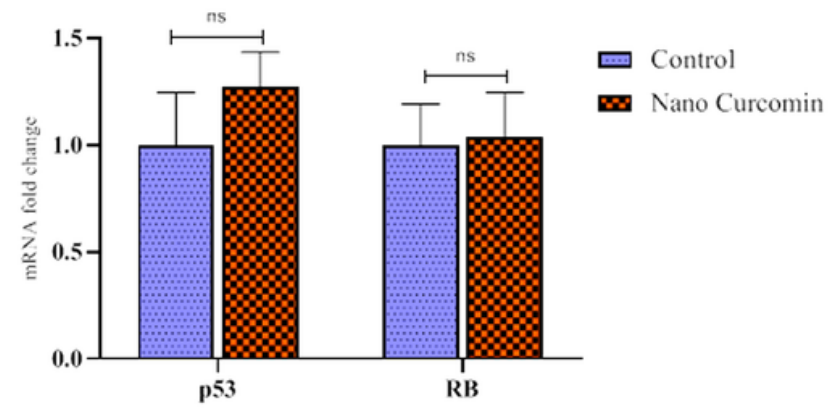

Figure 4

Expression analysis of E6, E7, P53 and Rb genes in cancerous HeLa cells and fibroblast cells by real time RT-PCR. (A \&B) A significant decrease in the expression of E6 and E7 genes and increase in the expression of P53 and Rb genes were observed in Hela cells. (C) No significant variation was occurred by nanocurcumin treatment of Rb and P53 genes expression in normal fibroblast cells (significant at * $\mathrm{p}$ value $<0.05,{ }^{\star \star} p$-value $<0.01,{ }^{* \star *}$-value $<0.001,{ }^{\star * \star *}$-value $\left.<0.0001\right)$. 
A
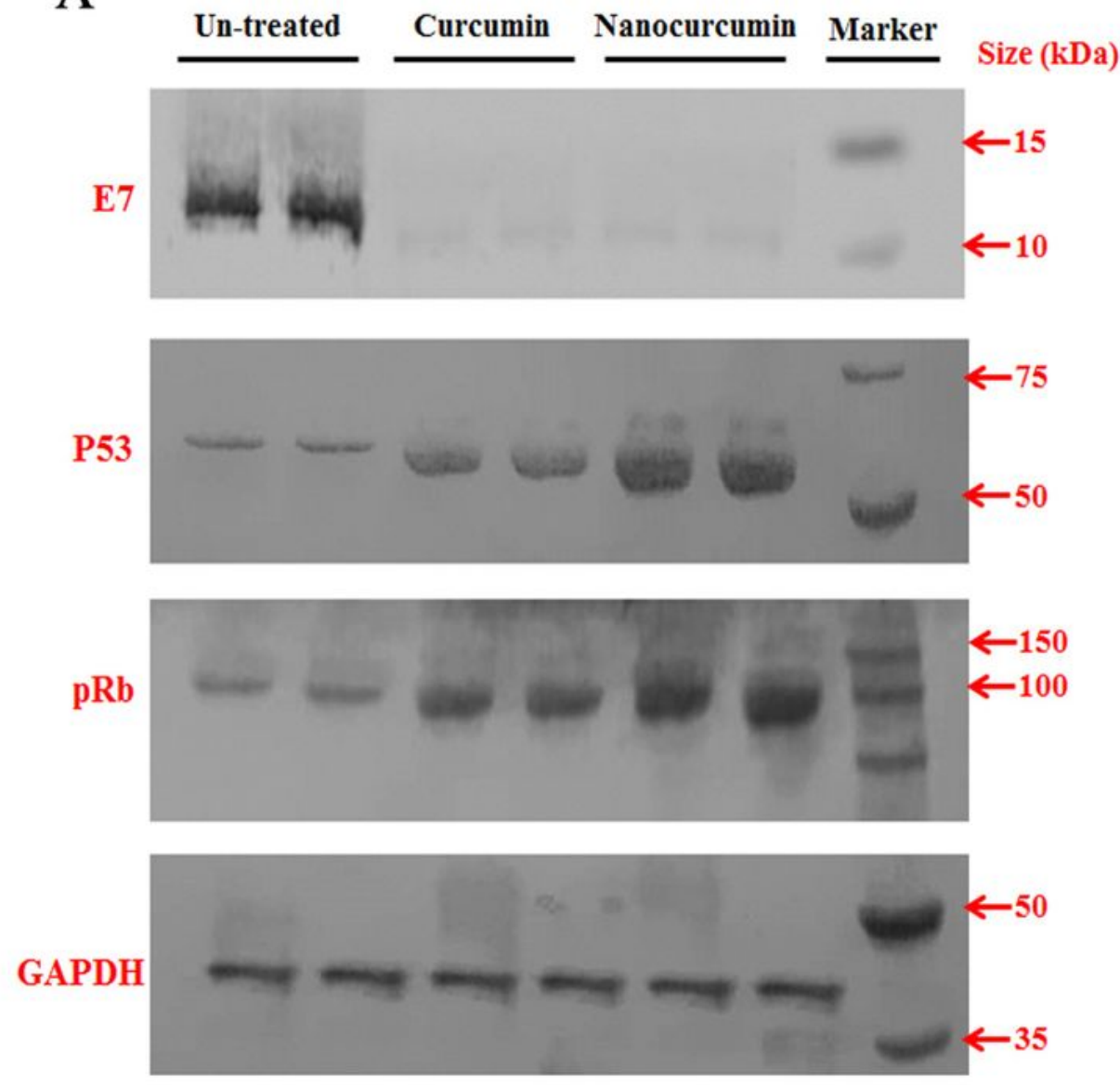

B
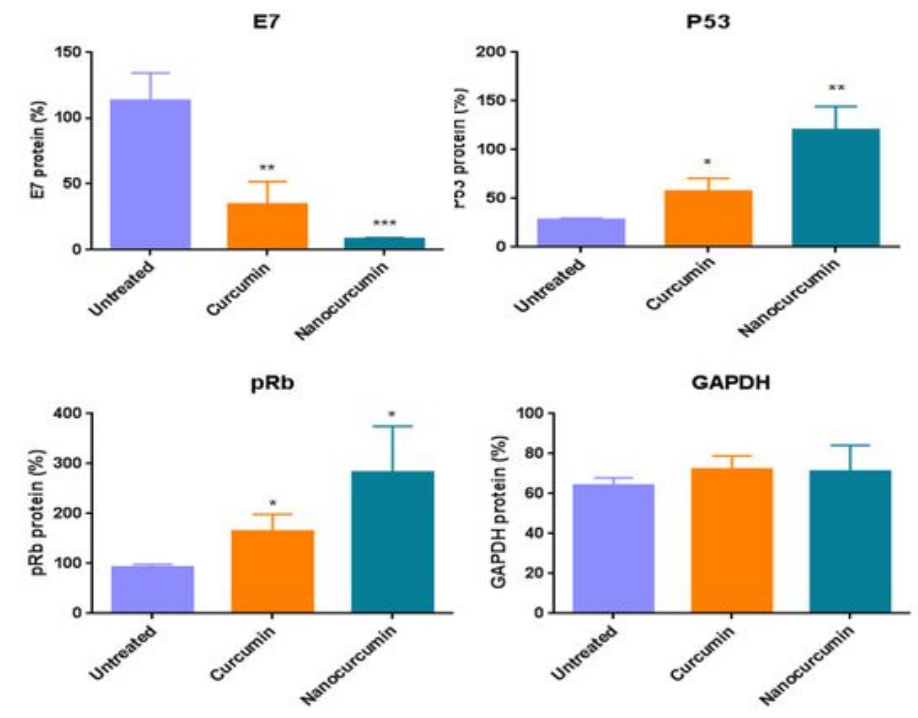

\section{Figure 5}

Western blotting results of E7, P53, pRb proteins and GAPDH protein (as control) in nanocurcumin and curcumin treated HeLa cells. (A) Nanocurcumin and curcumin treatment can decrease E7 protein expression and increase P53 and pRb proteins in cancerous HeLa cells. (B) Data has quantified by Image J software and the significant of protein alterations was determined (significant at ${ }^{*} \mathrm{p}$-value $<0.05$, ${ }^{* *}$ p-value $<0.01,{ }^{* \star *}$-value $\left.<0.001\right)$. 